\title{
Effects of Intranasal Cocaine on Sympathetic Nerve Discharge in Humans
}

\author{
Tage N. Jacobsen, Paul A. Grayburn, Richard W. Snyder II, Jim Hansen, Bahman Chavoshan, Charles Landau, \\ Richard A. Lange, L. David Hillis, and Ronald G. Victor \\ Department of Internal Medicine, Division of Cardiology, University of Texas Southwestern Medical Center, Dallas, Texas 75235-8573
}

\begin{abstract}
Cocaine-induced cardiovascular emergencies are mediated by excessive adrenergic stimulation. Animal studies suggest that cocaine not only blocks norepinephrine reuptake peripherally but also inhibits the baroreceptors, thereby reflexively increasing sympathetic nerve discharge. However, the effect of cocaine on sympathetic nerve discharge in humans is unknown. In 12 healthy volunteers, we recorded blood pressure and sympathetic nerve discharge to the skeletal muscle vasculature using intraneural microelectrodes (peroneal nerve) during intranasal cocaine $(2 \mathrm{mg} / \mathrm{kg}, n=8)$ or lidocaine $(2 \%, n=4)$, an internal local anesthetic control, or intravenous phenylephrine $(0.5-2.0 \mu \mathrm{g} / \mathrm{kg}, n=4)$, an internal sympathomimetic control. Experiments were repeated while minimizing the cocaine-induced rise in blood pressure with intravenous nitroprusside to negate sinoaortic baroreceptor stimulation. After lidocaine, blood pressure and sympathetic nerve discharge were unchanged. After cocaine, blood pressure increased abruptly and remained elevated for 60 min while sympathetic nerve discharge initially was unchanged and then decreased progressively over $60 \mathrm{~min}$ to a nadir that was only $2 \pm 1 \%$ of baseline $(P<0.05)$; however, plasma venous norepinephrine concentrations $(n=5)$ were unchanged up to $60 \mathrm{~min}$ after cocaine. Sympathetic nerve discharge fell more rapidly but to the same nadir when blood pressure was increased similarly with phenylephrine. When the cocaine-induced increase in blood pressure was minimized (nitroprusside), sympathetic nerve discharge did not decrease but rather increased by 2.9 times over baseline $(P<0.05)$. Baroreflex gain was comparable before and after cocaine.

We conclude that in conscious humans the primary effect of intranasal cocaine is to increase sympathetic nerve discharge to the skeletal muscle bed. Furthermore, sinoaortic baroreflexes play a pivotal role in modulating the cocaineinduced sympathetic excitation. The interplay between these excitatory and inhibitory neural influences determines the net effect of cocaine on sympathetic discharge targeted to
\end{abstract}

Address correspondence to Ronald G. Victor, M.D., Molecular Cardiology Laboratories, Division of Cardiology, Department of Internal Medicine, University of Texas Southwestern Medical Center, 5323 Harry Hines Blvd., NB11.120, Dallas, TX 75235-8573. Phone: 214-648-1406; FAX: 214-648-1450; E-mail: victor@ryburn.swmed.edu

Received for publication 11 April 1996 and accepted in revised form 6 December 1996.

J. Clin. Invest.

(C) The American Society for Clinical Investigation, Inc.

0021-9738/97/02/0628/07 \$2.00

Volume 99, Number 4, February 1997, 628-634 the human skeletal muscle circulation. (J. Clin. Invest. 1997. 99:628-634.) Key words: sympathetic nervous system • cocaine $\cdot$ baroreceptors $\bullet$ microelectrodes

\section{Introduction}

Cocaine abuse is a major cause of life-threatening cardiovascular emergencies including ventricular arrhythmias, acute myocardial infarcts, and hypertensive crises (1-5). Although there is evidence that all these emergencies are caused by excessive adrenergic stimulation of the cardiovascular system (6-10), the underlying mechanisms mediating cocaine's sympathomimetic effects are poorly understood.

The standard explanation is that cocaine blocks the norepinephrine reuptake transporter in peripheral sympathetic nerve terminals, thereby increasing norepinephrine concentration in the synaptic cleft (11-15). However, additional mechanisms must be involved, since other drugs (e.g., tricyclic antidepressants) which are more effective than cocaine at blocking the norepinephrine transporter do not cause the same cardiovascular catastrophic events (16). Data from experimental animals indicate that cocaine also modulates sympathetic nerve discharge, the neural stimulus to norepinephrine release, but there is no consensus as to how this occurs. Two conflicting hypotheses have been proposed. The first is that cocaine decreases sympathetic discharge centrally (17-19), which would mitigate the sympathomimetic effects of cocaine. The second hypothesis is that cocaine increases sympathetic discharge either centrally $(20,21)$ or reflexively, the latter by exerting a local anesthetic effect on the sinoaortic baroreceptors $(22,23)$. Increased sympathetic nerve discharge would amplify the peripheral sympathomimetic actions of cocaine. The evidence to support these hypotheses is derived mainly from studies in anesthetized, decerebrate, or ex vivo animal preparations and the effect of cocaine on sympathetic nerve discharge in conscious humans is unknown.

Accordingly, this study was undertaken to delineate the effect of cocaine on sympathetic nerve discharge in humans. Specifically, we asked two questions. First, does cocaine increase or decrease sympathetic nerve discharge? Second, does the sinoaortic baroreflex play a pivotal role in mediating the sympathetic nerve response to cocaine? To address these questions, we recorded postganglionic sympathetic action potentials targeted to the skeletal muscle vasculature in healthy volunteer subjects using microelectrodes inserted into fascicles of the peroneal nerve during the intranasal administration of cocaine.

\section{Methods}

\section{General methods}

We studied 14 healthy male volunteers, 24-44 yr of age. Microneurographic recordings of sympathetic nerve discharge were performed in 12 of the 14 subjects. The protocol was approved by the Institutional 
Review Board of the University of Texas Southwestern Medical Center, and all subjects gave their written informed consent to participate. All subjects were normotensive and had no history of cardiovascular disease. None of the subjects had any history of cocaine abuse or of other recreational drug abuse. None of the subjects were taking any prescription or nonprescription drugs with cardiovascular or autonomic effects.

All experiments were performed with the subjects in the supine position. Heart rate (electrocardiography), blood pressure (Finapres; Ohmeda, Madison, WI), and postganglionic efferent sympathetic nerve discharge targeted to the skeletal muscle circulation were recorded continuously using an electrostatic recorder (ES1000; Gould Inc., Glen Burnie, MD).

\section{Sympathetic nerve discharge}

Multiunit recordings of postganglionic sympathetic nerve discharge were obtained with unipolar tungsten microelectrodes inserted selectively into muscle nerve fascicles of the peroneal nerve posterior to the fibular head according to the technique of Vallbo et al. (24). Briefly, the neural signals were amplified 20,000-50,000 times, filtered (bandwidth, 700-2,000 Hz), rectified, and integrated (time constant, $0.1 \mathrm{~s}$ ) to obtain a mean voltage display of sympathetic discharge. A recording of sympathetic nerve discharge was considered acceptable when the neurograms revealed spontaneous, pulse synchronous bursts of neural activity, with the largest bursts showing a minimal signal-to-noise ratio of 3:1. In each experiment, we documented that we were recording sympathetic nerve discharge targeted to the skeletal muscle circulation by demonstrating that the neural activity had ( $a$ ) no response to arousal stimuli (loud noise or skin pinch) and $(b)$ a characteristic cardiac rhythmicity (i.e., the interburst interval was equal to or a whole number multiple of the cardiac cycle length). Sympathetic bursts were detected by inspection of the filtered and mean voltage neurograms. A deflection on the mean voltage display was counted as a burst if it had a minimal signal-to-noise ratio of 2:1. The interobserver and intraobserver variabilities in identifying bursts are $<10$ and $5 \%$, respectively (25). Inadvertent contraction of the leg muscles adjacent to the recording electrode produces electromyographic artifacts that are easily distinguished from sympathetic bursts; neurograms that revealed such artifacts were excluded from analysis. Nerve traffic was measured as the burst frequency times mean burst amplitude, an index of total nerve traffic.

\section{Echocardiography}

Two-dimensional echocardiography was performed in each subject using a $3.25 \mathrm{MHz}$ annular array transducer (CFM 750; Vingmed, Milpitas, CA). All studies were recorded on videotape for subsequent analysis by an experienced observer who was blinded to the intervention. Left ventricular end-diastolic and end-systolic dimensions and cross-sectional areas were measured using a parasternal short axis view at the midventricular level (26). Fractional area shortening was calculated as LVDed - LVDes/LVDed and LVAed - LVAed/LVAed, where LVD is the left ventricular dimension, LVA is left ventricular area, es is end-systole, and ed is end-diastole.

\section{Plasma norepinephrine levels}

Venous blood samples were obtained for analysis of norepinephrine concentrations from an indwelling cannula in a forearm vein. The samples were collected in chilled, heparinized tubes and promptly centrifuged at $4^{\circ} \mathrm{C}$ and then rapidly frozen. Norepinephrine concentrations were assayed using high performance liquid chromatography with an electrochemical detector (27) (SmithKline Beecham Clinical Laboratories, St. Louis, MO); this assay was sensitive to $10 \mathrm{pg} / \mathrm{ml}$ with a coefficient of variation of $10 \%$.

\section{Experimental protocols}

Protocol 1: Sympathetic nerve responses to intranasal cocaine versus intranasal lidocaine. After stable baseline data were obtained for 15 min, each subject was randomly assigned to receive intranasal $(a)$ co- caine hydrochloride, $2 \mathrm{mg} / \mathrm{kg}$ in a $10 \%$ solution $(n=8)$ or $(b)$ lidocaine hydrochloride, $2 \%$ solution $(n=4)$, the latter used to control for the local anesthetic property of cocaine. Each solution $(\sim 10 \mathrm{ml})$ was administered slowly to both nares over $10 \mathrm{~min}$. This dose of intranasal cocaine is half the standard clinical dose for rhinolaryngologic procedures (28). Heart rate, blood pressure, and sympathetic nerve discharge were recorded continuously for $90 \mathrm{~min}$. At the end of the 90-min measurement, the stability of the microneurographic recording site was verified by the ability of a Valsalva maneuver to evoke a characteristically large increase in sympathetic nerve discharge.

To study the underlying mechanisms mediating the observed changes in sympathetic nerve discharge after cocaine, additional protocols were performed on separate days using the same subjects.

Protocol 2: Sympathetic nerve responses to cocaine versus dobutamine. Cocaine increases ventricular contractility (as reflected by left ventricular fractional area shortening), which would be expected to increase the mechanical stimulation of inhibitory left ventricular mechanoreceptor afferents (29). Therefore, the aim of this protocol was to examine the role played by cardiac mechanoreceptor stimulation in mediating the changes in sympathetic discharge elicited by cocaine. To accomplish this, we compared the sympathetic nerve responses to cocaine with those evoked by dobutamine, used as an internal control for the positive inotropic action of cocaine. In four subjects, measurements of sympathetic nerve discharge, blood pressure, heart rate, and left ventricular fractional area shortening were repeated on a separate day as dobutamine was infused intravenously for $60 \mathrm{~min}$ with the dose being titrated $(5.0-10.0 \mu \mathrm{g} / \mathrm{kg} / \mathrm{min})$ to match the increases in left ventricular fractional area shortening produced previously by the cocaine.

Protocol 3: Sympathetic nerve responses to cocaine versus phenylephrine. Cocaine increases arterial pressure, the stimulus to the sinoaortic baroreceptors (30). Therefore, this protocol was designed to examine the role played by sinoaortic baroreceptor stimulation in mediating the changes in sympathetic discharge elicited by cocaine. To accomplish this, we compared the sympathetic nerve responses to cocaine with those evoked by phenylephrine, a direct sympathomimetic agent used as an internal control for the blood pressure-raising action of cocaine. In four subjects, measurements of arterial pressure, heart rate, and sympathetic nerve discharge were repeated on a separate day as phenylephrine hydrochloride was infused intravenously in $15 \mathrm{~min}$, with the dose $(1.0-2.5 \mu \mathrm{g} / \mathrm{kg} / \mathrm{min})$ being titrated to produce increases in mean arterial pressure which mimicked the cocaineinduced increases in both time course and magnitude.

Protocol 4: Effects of pharmacologic attenuation of the cocaineinduced elevation in blood pressure on sympathetic nerve responses to cocaine. This protocol was designed to minimize sinoaortic baroreceptor stimulation during cocaine by attenuating the cocaine-induced increase in blood pressure with intravenous sodium nitroprusside (31). In four subjects, we recorded sympathetic nerve discharge for 60 min after intranasal cocaine while simultaneously infusing sodium nitroprusside. The nitroprusside infusion was started immediately during the intranasal administration of cocaine and the dose of nitroprusside $(1.0-3.0 \mu \mathrm{g} / \mathrm{kg} / \mathrm{min})$ was titrated individually to attenuate the normal cocaine-induced increase in blood pressure by at least $50 \%$ (i.e., by $6-8 \mathrm{mmHg}$ ). During the infusion of nitroprusside, particular care was taken not to lower blood pressure below the resting baseline value. In the same four subjects, on a separate day we tested the effects of nitroprusside on the reflex decreases in sympathetic nerve discharge elicited by phenylephrine, used as a direct sympathomimetic control; the dose of nitroprusside was titrated to attenuate the phenylephrine-induced increases in blood pressure by $\sim 50 \%(6-8$ $\mathrm{mmHg}$ ).

Protocol 5: Effects of intranasal cocaine on the gain of the sinoaortic baroreflex. To quantify sinoaortic baroreflex gain in the presence or absence of cocaine, we measured progressive reflex increases in sympathetic nerve discharge evoked by progressive decreases in arterial pressure during the intravenous infusion of sodium nitroprusside, 


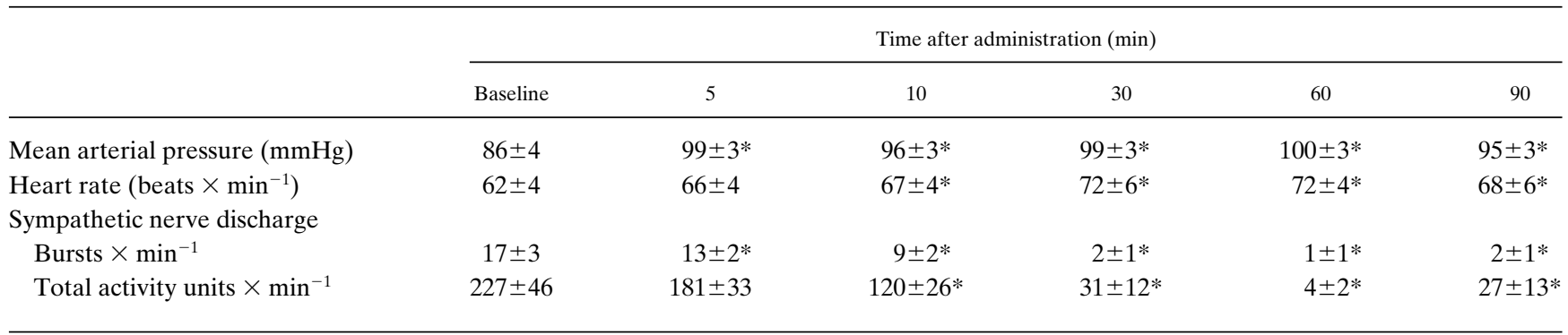

Data are mean $\pm \mathrm{SE}$ for eight subjects. $* P<0.05$ vs. baseline.

with the dose titrated to decrease mean arterial pressure progressively to a value of $\sim 15 \mathrm{mmHg}$ below baseline. Baroreflex gain, measured before and again $30 \mathrm{~min}$ after cocaine, was calculated as the increase in sympathetic discharge per $\mathrm{mmHg}$ decrease in mean arterial pressure.

Protocol 6: Effects of intranasal cocaine on plasma venous norepinephrine concentrations. To examine the net effects of the various central neural and peripheral neuroeffector actions of cocaine on plasma norepinephrine concentrations, in five subjects (including three subjects who had undergone microneurography and two who had not), 10-ml aliquots of venous blood were withdrawn at 60, 20, and $0 \mathrm{~min}$ before cocaine and at 20 and $60 \mathrm{~min}$ after intranasal cocaine $(2 \mathrm{mg} / \mathrm{kg})$. The three separate baseline measurements were performed to document the stability of plasma norepinephrine concentrations after insertion of the venous cannula.

\section{Statistical methods}

All data are expressed as mean \pm 1 SEM. All statistical analyses were performed using a repeated measures ANOVA with Scheffe's posthoc test for multiple comparisons over time. $P<0.05$ was considered statistically significant.

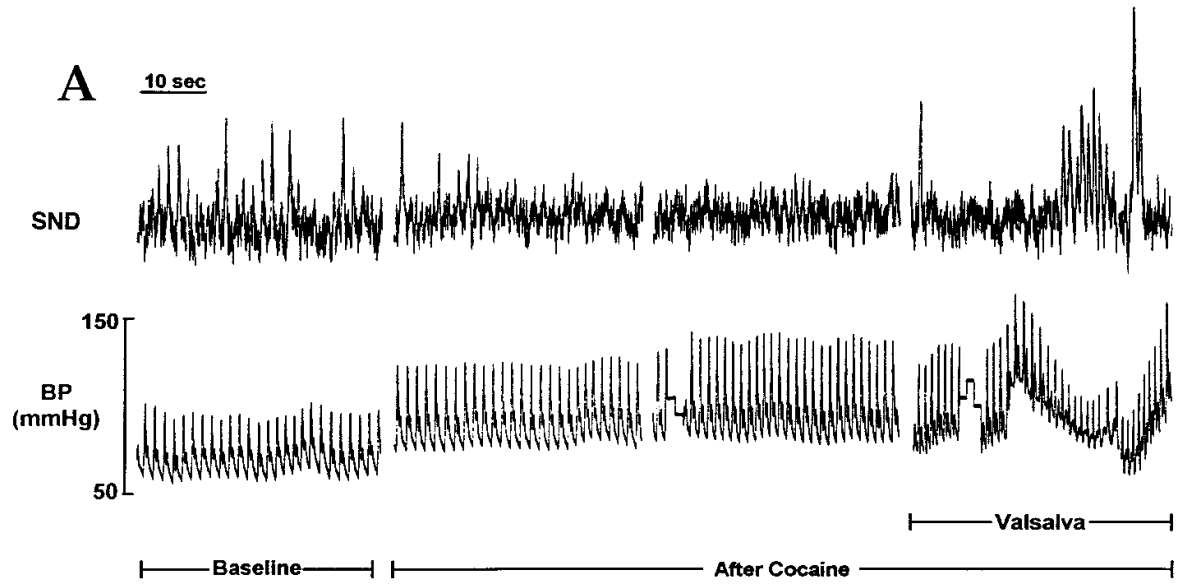

B

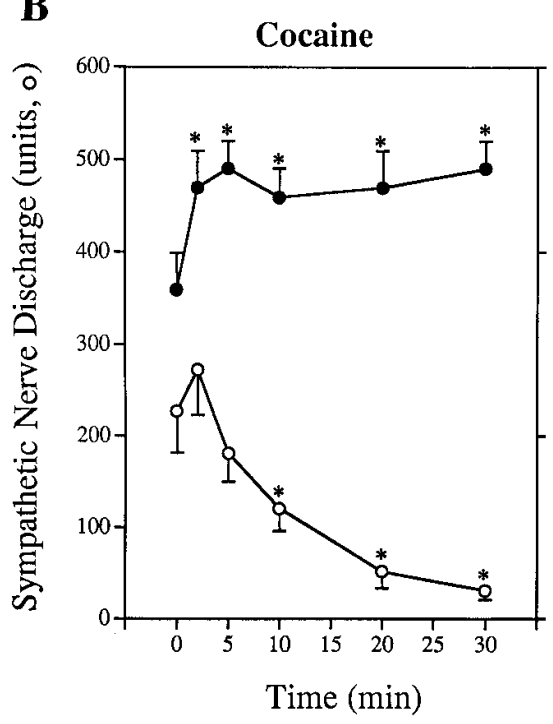

Figure 1. Effects of intranasal cocaine on blood pressure and sympathetic nerve discharge. (A) Segments of an illustrative experiment in one subject showing recordings of sympathetic nerve discharge and blood pressure at baseline and $30 \mathrm{~min}$ after intranasal cocaine. The sympathetic discharge is proportional to the frequency and the amplitude of the spikes (bursts). After cocaine, sympathetic nerve discharge decreased as blood pressure increased. Nevertheless, transient increases in sympathetic discharge still could be elicited by performance of a Valsalva maneuver, documenting the stability of the microelectrode recording and preservation of baroreflex function after cocaine. (B) Summary data showing changes in sympathetic nerve discharge and blood pressure plotted as a function of time after intranasal administration of cocaine (eight subjects) or during intravenous infusion of the direct alpha adrenergic agonist phenylephrine (four subjects), the latter titrated to mimic the blood pressure response to cocaine. Data are mean \pm SE. $* P<0.05$ vs. baseline (time 0 ). For comparable increases in blood pressure, sympathetic nerve discharge decreased more slowly with cocaine than with phenylephrine. 
Table II. Responses to Intransal Lidocaine

\begin{tabular}{lcccc}
\hline & \multicolumn{4}{c}{ Time after administration (min) } \\
\cline { 2 - 5 } & Baseline & 5 & 10 & 30 \\
\hline Mean arterial pressure $(\mathrm{mmHg})$ & $86 \pm 3$ & $86 \pm 2$ & $87 \pm 3$ & $87 \pm 3$ \\
Heart rate (beats $\times$ min $\left.^{-1}\right)$ & $53 \pm 4$ & $54 \pm 4$ & $52 \pm 6$ & $55 \pm 4$ \\
Sympathetic nerve discharge & & & & \\
$\quad$ Bursts $\times$ min $^{-1}$ & $17 \pm 4$ & $18 \pm 4$ & $17 \pm 3$ & $18 \pm 4$ \\
$\quad$ Total activity units $\times \min ^{-1}$ & $274 \pm 48$ & $301 \pm 56$ & $265 \pm 35$ & $291 \pm 58$
\end{tabular}

Data are mean \pm SE for four subjects.

\section{Results}

None of the subjects developed chest pain or any electrocardiographic evidence of myocardial ischemia or arrhythmias, or other complications from cocaine. After intranasal cocaine, blood pressure and heart rate, as expected, increased abruptly, remained elevated for $60 \mathrm{~min}$, and thereafter began to return toward baseline (Table I and Fig. 1). These increases in heart rate and blood pressure (the latter measured by Finapres) were comparable with those reported previously using central aortic catheterization to measure blood pressure after the same dose of intranasal cocaine $(7,32,33)$. Sympathetic nerve discharge at first was unchanged despite increased blood pressure and then decreased progressively over $60 \mathrm{~min}$ to a nadir that was $2 \pm 1 \%$ of the baseline value $(227 \pm 46$ to $4 \pm 2 \mathrm{U}$; $P<0.05$ ) (Table I and Fig. 1). In contrast to intranasal cocaine, intranasal lidocaine had no effects on blood pressure, heart rate, or sympathetic nerve discharge (Table II). Although dobutamine produced increases in left ventricular fractional shortening comparable with those produced by cocaine $(0.54 \pm 0.04$ to $0.67 \pm 0.02$ vs. $0.54 \pm 0.03$ to $0.63 \pm 0.03$, dobutamine vs. cocaine), dobutamine had no effect on heart rate ( $56 \pm 4$ beats/min at baseline vs. $60 \pm 4$ beats/min after dobutamine), mean arterial pressure ( $88 \pm 2$ vs. $90 \pm 2 \mathrm{mmHg}$ ), or sympathetic nerve discharge ( $13 \pm 2$ vs. $16 \pm 3$ bursts/min; $230 \pm 29$ vs. $266 \pm 29$ total activity units).

Despite comparable increases in blood pressure, sympathetic nerve discharge decreased more slowly with cocaine than with phenylephrine (Fig. 1), although the peak decreases in sympathetic discharge were comparable with both drugs. When the cocaine-induced elevation in blood pressure was attenuated by $66 \%$ (with nitroprusside), sympathetic nerve discharge did not decrease (as with cocaine alone) but rather increased markedly to a peak value that was 2.9 times higher than baseline $(217 \pm 63$ to $639 \pm 137$ activity units, $P<0.05)$. (Fig. 2 and Table III). This increase in sympathetic discharge peaked within the first 5 min after administration of cocaine and over the next 60 min declined partially to a value that was 1.9 times higher than baseline. When the nitroprusside infusion was discontinued, sympathetic nerve discharge decreased abruptly as blood pressure was allowed to increase normally in response to cocaine (Fig. 2). In contrast, when nitroprusside was used similarly to attenuate a phenylephrine-induced elevation in blood pressure, sympathetic nerve discharge did not increase above the baseline (Fig. 2).

In all subjects, the characteristic biphasic sympathetic nerve response to Valsalva's maneuver was preserved after cocaine (Fig. 1). The gain of the sinoaortic baroreflex was comparable before versus after cocaine: $69 \pm 24$ vs. $60 \pm 17 \mathrm{U} /$ $\mathrm{mmHg}(P=\mathrm{NS})$.

Plasma norepinephrine concentrations were unchanged after intranasal cocaine. The values in picograms per milliliter were $187 \pm 39,174 \pm 29$, and $191 \pm 28$ at 60,20 , and $0 \mathrm{~min}$, respectively, before cocaine and were $190 \pm 28$ and $210 \pm 15$ at 20 and $60 \mathrm{~min}$, respectively, after cocaine; there were no significant differences between any of these values by ANOVA $(P=\mathrm{NS})$.

\section{Discussion}

An emerging body of experimental animal literature indicates that cocaine, in addition to blocking norepinephrine reuptake peripherally, can exert two directionally opposite effects on central sympathetic outflow: (a) sympathetic inhibition due to interruption of excitatory neural circuits in the brain (17-19) and $(b)$ sympathetic excitation due to either a direct central neural action $(20,21)$ or inhibition of sinoaortic baroreceptor reflexes $(22,23)$. The ability to measure sympathetic nerve discharge directly with intraneural microelectrodes (24) provided the opportunity to test these different theories in humans. The major new findings are twofold. First, in conscious humans the primary effect of cocaine is to increase, not decrease, sympathetic nerve discharge to the skeletal muscle bed. Second, sinoaortic baroreflexes are not attenuated by cocaine but rather play a pivotal role in modulating the cocaine-induced sympathetic excitation. The interplay between these excitatory and inhibitory neural influences determines the net effect of cocaine on sympathetic discharge targeted to the human peripheral circulation.

In our healthy subjects, the net effect of intranasal cocaine was a decrease in sympathetic nerve discharge. At first glance, this result would appear to be consistent with animal studies

Table III. Responses to Intranasal Cocaine during Infusion of Nitroprusside

\begin{tabular}{|c|c|c|c|c|c|}
\hline & \multicolumn{5}{|c|}{ Time after administration (min) } \\
\hline & Baseline & 5 & 10 & 30 & 60 \\
\hline Mean arterial pressure $(\mathrm{mmHg})$ & $94 \pm 12$ & $100 \pm 11$ & $97 \pm 11$ & $97 \pm 10$ & $99 \pm 9$ \\
\hline Heart rate $\left(\min ^{-1}\right)$ & $58 \pm 5$ & $70 \pm 7 *$ & $68 \pm 6^{*}$ & $72 \pm 6^{*}$ & $72 \pm 7 *$ \\
\hline \multicolumn{6}{|l|}{ Sympathetic nerve discharge } \\
\hline Bursts $\times \min ^{-1}$ & $11 \pm 3$ & $25 \pm 5 *$ & $24 \pm 7 *$ & $23 \pm 7 *$ & $18 \pm 5^{*}$ \\
\hline Total activity units $\times \min ^{-1}$ & $217 \pm 63$ & $639 \pm 137 *$ & $551 \pm 117 *$ & $559 \pm 259 *$ & $418 \pm 143 *$ \\
\hline
\end{tabular}

Data are mean \pm SE for four subjects. $* P<0.05$ vs. baseline. 

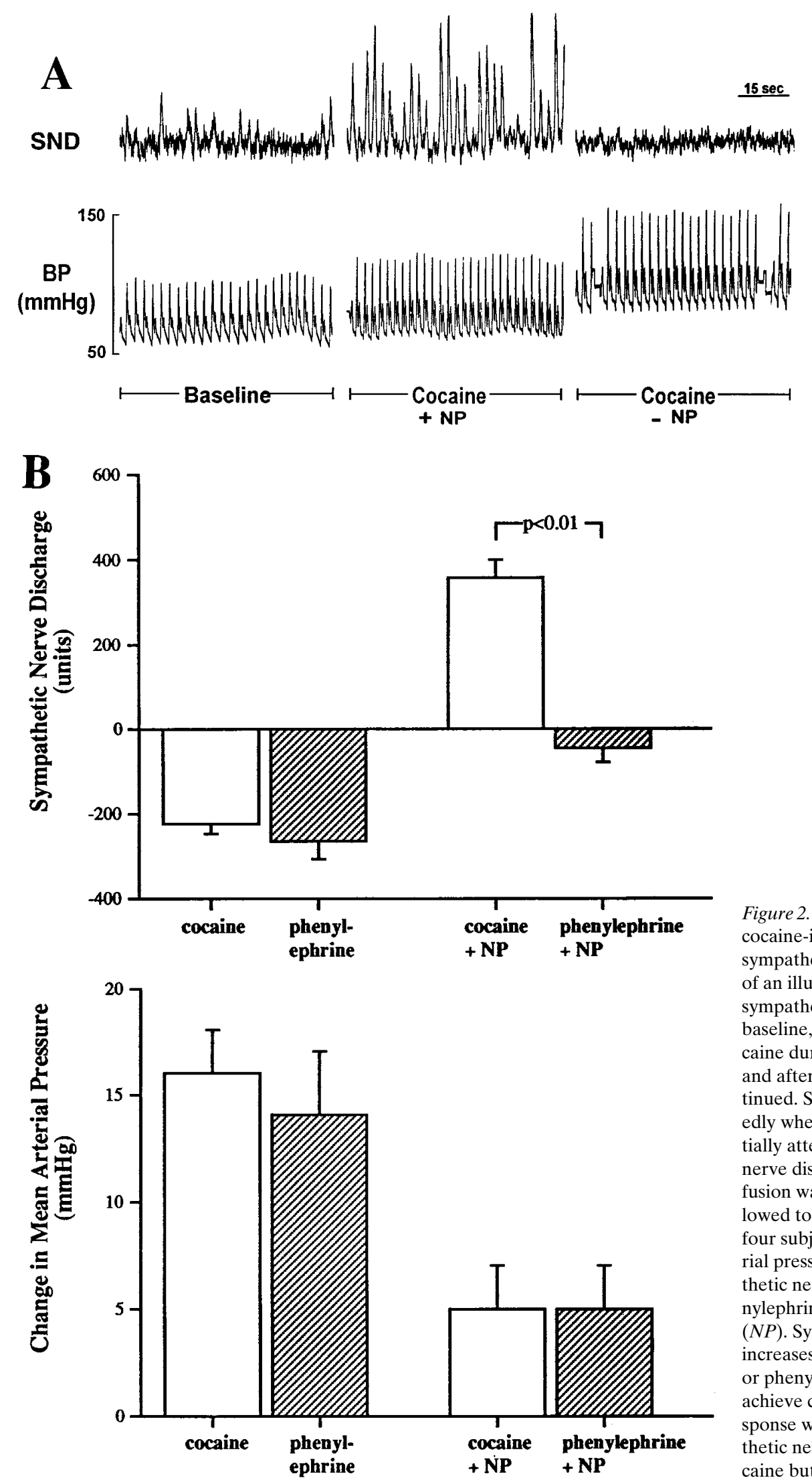

Figure 2. Effects of pharmacologic suppression of the cocaine-induced increase in blood pressure on the sympathetic nerve response to cocaine. ( $A$ ) Segments of an illustrative experiment in one subject showing sympathetic nerve discharge and blood pressure at baseline, 30 min after intranasal administration of cocaine during intravenous infusion of nitroprusside, and after the nitroprusside infusion had been discontinued. Sympathetic nerve discharge increased markedly when the pressor response to cocaine was partially attenuated with nitroprusside. Sympathetic nerve discharge decreased when the nitroprusside infusion was discontinued and blood pressure was allowed to increase. (B) Summary data (mean \pm SE) in four subjects showing the peak changes in mean arterial pressure and corresponding changes in sympathetic nerve discharge elicited by cocaine versus phenylephrine alone or in combination with nitroprusside $(N P)$. Sympathetic nerve discharge decreased during increases in blood pressure evoked by either cocaine or phenylephrine. When nitroprusside was used to achieve comparable attenuations in the pressor response with either sympathomimetic drug, sympathetic nerve discharge increased markedly with cocaine but did not increase with phenylephrine. 
demonstrating that cocaine decreases sympathetic discharge centrally (17-19). Alternatively, the decreased sympathetic discharge might be a reflex response (rather than a centrally mediated response) evoked by activation of either $(a)$ sinoaortic baroreceptors attendant to the cocaine-induced increase in blood pressure (30) or $(b)$ cardiac ventricular mechanoreceptors attendant to the cocaine-induced increase in ventricular contractility (29). We readily excluded the latter explanation because in the same subjects sympathetic nerve discharge was unaffected when we mimicked the cocaine-induced increase in left ventricular fractional area shortening with dobutamine.

On the other hand, the data implicate a pivotal role for the sinoaortic baroreflex in modulating the sympathetic nerve response to intranasal cocaine. Sinoaortic baroreflex activation explains the decreased sympathetic discharge after cocaine because this decrease was approximated when we mimicked the cocaine-induced rise in blood pressure with phenylephrine and it was eliminated (and even reversed) when we minimized the cocaine-induced rise in blood pressure using nitroprusside. That sympathetic nerve discharge fell more slowly with cocaine than with phenylephrine led us to suspect that cocaine exerts a primary excitatory effect on sympathetic nerve discharge, which initially offsets the inhibitory effect of baroreflex activation. We confirmed this suspicion when we unmasked a dramatic increase in sympathetic nerve discharge by clamping blood pressure (i.e., sinoaortic baroreflex activation) after cocaine. The evidence that this sympathetic excitation is a specific property of cocaine rather than a nonspecific property of any sympathomimetic agent is that the sympathetic discharge did not increase over baseline when we clamped blood pressure during infusion of phenylephrine. These findings are consistent with studies in rats suggesting that central sympathetic activation contributes to the acute blood pressure-raising effect of cocaine $(20,21)$.

From the present experiments, it would be difficult to explain cocaine-induced sympathetic excitation on the basis of an impaired baroreflex, as suggested by previous animal studies $(22,23)$. Those studies advanced the concept that cocaine anesthetizes the baroreceptors, a lidocaine-like effect, thereby reflexively increasing efferent sympathetic nerve discharge and blood pressure. However, the evidence for that concept was derived from direct application of cocaine (or lidocaine) to the baroreceptor nerve endings. In human subjects, however, it is unlikely that baroreceptor function would be affected by the intranasal administration of the relatively small dose of cocaine used in our study because of dilution in the systemic circulation. Indeed, in the present experiments, there was no detectable effect of cocaine on baroreflex control of sympathetic nerve discharge. After cocaine, sympathetic discharge increased normally in response to the Valsalva maneuver, a standard but qualitative test of baroreflex function. Baroreflex gain, quantified as the reflex increase in sympathetic discharge produced by a given decrease in arterial pressure, was equivalent before and after intranasal cocaine. Sympathetic nerve discharge was unaffected by intranasal administration of lidocaine, another local anesthetic agent. The latter finding is consistent with previous data indicating that even an antiarrhythmic dose of intravenous lidocaine has little (34) or no (35) effect on baroreflex control of muscle sympathetic nerve discharge. Although these findings certainly do not prove that intranasal cocaine has absolutely no effect on sinoaortic baroreflexes in humans, the data suggest that cocaine does not cause a major impairment in the baroreflex control of muscle sympathetic nerve discharge.

Thus, the principal new concept arising from these microneurographic data is that a baroreflex-mediated decrease in sympathetic nerve discharge overrides an excitatory action of intranasal cocaine on central sympathetic outflow. However, the main mechanism mediating the sympathomimetic actions of cocaine long has been thought to be blockade of norepinephrine reuptake in peripheral sympathetic nerve terminals (11-15). The additional finding that plasma norepinephrine concentrations were unchanged with intranasal cocaine suggests that the reflex decrease in postganglionic sympathetic nerve discharge is of sufficient magnitude to negate an expected increase in plasma norepinephrine resulting from inhibition of peripheral norepinephrine reuptake.

This interpretation is based on the intranasal administration of a dose of cocaine which is equivalent to one-half of a standard local anesthetic dose. From these experiments, the possibility remains that the balance between the various sympathoexcitatory and inhibitory actions of cocaine might differ substantially with higher doses of intranasal cocaine than used in this study or with other routes of administration (intravenous or freebase inhalation) which produce higher plasma cocaine concentrations.

Based on these experimental findings with a small dose of intranasal cocaine in healthy humans, we suggest that preservation of normal baroreflex function provides a neurophysiologic explanation for the surprisingly infrequent occurrence of cocaine-induced cardiovascular catastrophic events in the current epidemic of cocaine abuse. In the setting of recreational cocaine abuse, this protective reflex mechanism might be overcome by an excessively high dose of cocaine or it might be impaired in individuals with attenuated baroreflexes due to preexisting medical conditions such as essential hypertension.

\section{Acknowledgments}

This study was supported by grants to Dr. Jacobsen from The Danish Heart Foundation, Danish Research Academy, S\&W Foundation (Denmark), Leo's Kemiske Fabrik Research Foundation (Denmark), Nycomed-DAK (Denmark), and a Fogarty International Research Fellowship (NIH 1-F05-TW04494); to Dr. Hansen from the Danish Heart Foundation and a Fogarty International Research Fellowship (NIH 1-F05-TW04949); to Dr. Chavoshan from the National Heart, Lung, and Blood Institute (5T-32 HL07360-19); and to Dr. Victor from the National Institute on Drug Abuse (RO-1 DA10064).

\section{References}

1. Cregler, L.L., and H. Mark 1986. Medical complications of cocaine abuse. N. Engl. J. Med. 315:1495-1500.

2. Smith, H.W.B., III, H.A. Liberman, S.L. Brody, L.L. Battey, B.C. Donahue, and D.C. Morris. 1987. Acute myocardial infarction temporally related to cocaine use: clinical, angiographic, and pathophysiologic observations. Ann. Intern. Med. 107:13-18.

3. Isner, J.M., N.A.M. Estes, P.D. Thompson, M.R. Constanzo-Nordin, G. Miller, G. Katsas, K. Sweeney, and W.Q. Sturner. 1986. Acute cardiac events temporally related to cocaine abuse. N. Engl. J. Med. 315:1438-1443.

4. Kloner, R.A., S. Hale, K. Alker, and S. Rezkalla. 1992. The effects of acute and chronic cocaine use on the heart. Circulation. 85:407-419.

5. Warner, W.A. 1993. Cocaine abuse. Ann. Intern. Med. 119:226-235.

6. Resnick, R.B., R.S. Kestenbaum, and L.K. Schwartz. 1977. Acute systemic effects of cocaine in man: a controlled study by intranasal and intravenous routes. Science (Wash. DC). 195:696-698.

7. Lange, R.A., R.G. Cigarroa, C.W. Yancy, J.E. Willard, J.J. Popma, M.N. Sills, W. McBride, A.S. Kim, and L.D. Hillis. 1989. Cocaine-induced coronary artery vasoconstriction. N. Engl. J. Med. 321:1557-1562. 
8. Lange, R.A., and J.E. Willard. 1993. The cardiovascular effects of cocaine. Heart Dis. Stroke. 2:136-141.

9. Karch, S.B., and M.E. Billingham. 1988. The pathology and etiology of cocaine-induced heart disease. Arch. Pathol. Lab. Med. 112:225-230.

10. Gradman, A.H. 1988. Cardiac effects of cocaine: a review. Yale J. Biol. Med. 61:137-147.

11. Whitby, L.G., G. Herting, and J. Axelrod. 1960. Effects of cocaine on the disposition of noradrenalin labeled with tritium. Nature (Lond.). 187:604605 .

12. Muscholl, E. 1961. Effects of cocaine and related drugs on the uptake of noradrenalin by heart and spleen. Br. J. Pharmacol. 16:352-359.

13. Furchgott, R.F., S.M. Kirpeker, M. Rieker, and A. Schwab. 1963. Action and interactions of norepinephrine, tyramine and cocaine on aortic strips of rabbit and left atria guinea pig and cat. J. Pharmacol. 142:39-58.

14. Jaffe, J.H. 1990. Drug addiction and drug abuse. In The Pharmacologic Basis of Therapeutics. A.G. Gilman, T.W. Rall, A.S. Nies, and P. Taylor, editors. Pergamon Press, New York. 539-546.

15. Gillis, R.A., Y.M. Hernandez, H.K. Erzouki, F.C. Rackowski, A.K. Mandal, F.E. Kuhn, and K.L. Dretchen. 1995. Sympathetic nervous system mediated cardiovascular effects of cocaine are primarily due to a peripheral site of action of the drug. Drug Alcohol Depend. 37:217-230.

16. Schroeder, J.S., A.V. Mullin, G.R. Elliot, H. Steiner, M. Nichols, A. Gordon, and M. Paulos. 1989. Cardiovascular effects of desipramine in children. J. Am. Acad. Child Adolesc. Psychiatry. 28:376-379.

17. Raczkowski, V.F.C., Y.M. Hernandez, H.K. Erzouki, T.P. Abrahams, A.K. Mandal, P. Hamosh, E. Friedman, J.A. Quest, K.L. Dretchen, and R.A. Gillis. 1991. Cocaine acts in the central nervous system to inhibit sympathetic neural activity. J. Pharmacol. Exp. Ther. 257:511-519.

18. Gantenberg, N.S., and G.R. Hageman. 1991. Cocaine depresses cardiac sympathetic efferent activity in anesthetized dogs. J. Cardiovasc. Pharmacol. 17:434-439.

19. Hageman, G.R., and T. Simor. 1993. Attenuation of the cardiac effects of cocaine by dizocilpine. Am. J. Physiol. 264:H1890-H1895.

20. Knuepfer, M.M., and C.A. Branch. 1992. Cardiovascular responses to cocaine are initially mediated by the central nervous system. J. Pharmacol. Exp. Ther. 263:734-741.

21. Wilkerson, R.D. 1988. Cardiovascular effects of cocaine in conscious dogs: importance of fully functional autonomic and central nervous systems. $J$. Pharmacol. Exp. Ther. 246:466-471.

22. Andresen, M.C., M. Yang, S.H. Nelson, and O.S. Steinsland. 1990. Cocaine inhibits baroreflex control of arterial pressure by action at arterial barore- ceptor. Am. J. Physiol. 258:H1244-H1249.

23. Trouve, R., G. Nahas, and C. Latour. 1992. Inhibition by cocaine of the baroreflex in the rat. Proc. Soc. Exp. Biol. Med. 201:215-218.

24. Vallbo, A.B., K.-E. Hagbarth, A. Hongell, and B.G. Wallin. 1979. Somatosensory proprioceptive, and sympathetic activity in human muscle nerves. Physiol. Rev. 59:919-957.

25. Mark, A.L., R.G. Victor, C. Nerhed, and B.G. Wallin. 1985. Microneurographic studies of the mechanisms of sympathetic nerve responses to static exercise in humans. Circ. Res. 57:461-469.

26. Schiller, N.B., P.M. Shah, M. Crawford, A. DeMaria, R. Devereaux, H. Feigenbaum, H. Gutgesell, N. Reichek, D. Sahn, I. Schnittger, et al. 1989. Recommendations for quantitation of the left ventricle by two-dimensional echocardiography. J. Am. Soc. Echo. 2:358-367.

27. Goldstein, D.S., D. Feuerstein, J.L. Izzo, I.J. Kopin, and H.R. Keiser. 1981. Validity and reliability of liquid chromatography with electrochemical detection for measuring plasma levels of norepinephrine and epinephrine in man. Life Sci. 28:467-475.

28. Johns, M.E., and R.L. Henderson. 1977. Cocaine use by the otolaryngologist: a survey. Trans. Am. Acad. Opthalmol. Otolaryngol. 84:969-973.

29. Thoren, P. 1977. Characteristics of left ventricular receptors with nonmedullated vagal afferents in cats. Circ. Res. 40:415-421.

30. Brown, A.M. 1980. Receptors under pressure: an update on baroreceptors. Circ. Res. 46:1-10.

31. Scherrer, U., S.L. Pryor, L.A. Bertocci, and R.G. Victor. 1990. Arterial baroreflex buffering of sympathetic activation during exercise-induced elevations in arterial pressure. J. Clin. Invest. 86:1855-1861.

32. Brogan, W.C., R.A. Lange, A.S. Kim, D.J. Moliterno, and L.D. Hillis. 1991. Alleviation of cocaine-induced coronary vasoconstriction by nitroglycerin. J. Am. Coll. Cardiol. 18:581-586.

33. Lange, R.A., R.G. Cigarroa, E.D. Flores, W. McBride, A.S. Kim, P.J Wells, J.B. Bedotto, R.S. Danziger, and L.D. Hillis. 1990. Potentiation of cocaine induced vasoconstriction by beta-adrenergic blockade. Ann. Intern. Med. 112:897-903.

34. Ebert, T.J., P.K. Mohanty, and J.P. Kampine. 1991. Lidocaine attenuates efferent sympathetic responses to stress in humans. J. Cardiothor. Vasc. Anaes. 5:437-443.

35. Ellenbogen, K.A., M.L. Smith, L.A. Beightol, and D.L. Eckberg. 1992. Influence of lidocaine on human muscle sympathetic nerve activity during programmed electrical stimulation and ventricular tachycardia. Am. Heart J. 124: 891-897. 